\title{
Particularidades no manejo do diabetes em pacientes nefropatas
}

\author{
Raquel C. Abi-Abib ${ }^{1 *}$
}

\section{Resumo}

O diabetes é uma doença sistêmica que pode comprometer vários órgãos, com redução de qualidade e expectativa de vida. Dentre os órgãos afetados, a nefropatiadiabética merece destaque, sendo o diabetes a principal causa de doença renal terminal em nosso país e no mundo. O tratamento de pacientes diabéticos com insuficiência renal é um grande desafio clínico, pois a presença de nefropatia grave leva a alterações metabólicas que aumentam o risco de hipoglicemia. Há aumento da meia-vida da insulina em pacientes nefropatas. Já o desenvolvimento de novos hipoglicemiantes orais nos últimos anos ampliou as opções terapêuticas potencialmente vantajosas para esse grupo de pacientes. Por outro lado, a complexidade na individualização do tratamento torna-se ainda maior, pois é fundamental conhecer as contraindicações, ajustes de posologia necessários, riscos de efeitos colaterais e nefrotoxicidade de cada uma dessas drogas. Em pacientes em diálise, vários fatores podem prejudicar o controle glicêmico, incluindo tanto fatores relacionados à nefropatia quanto relacionados ao método dialítico empregado. Outro fator que contribui para a complexidade do tratamento desses pacientes é o fato dos parâmetros laboratoriais tradicionalmente utilizados para a avaliação do controle glicêmico sofrerem influências importantes em decorrência da doença renal terminal, podendo haver redução da sua acurácia. O conhecimento de todos esses fatores é fundamental para que o complexo tratamento dos pacientes diabéticos com doença renal terminal possa ser adequadamente individualizado, de modo a se obter o melhor controle glicêmico possível com o mínimo risco de hipoglicemia.

Descritores: Insuficiência renal; Diabetes mellitus; Terapêutica.

\section{Abstract \\ Particularities in managing diabetes in nephro- pathic patients}

Diabetes is a systemic disease that can cause damage to various organs, leading to reduction in quality of life and life expectancy. Among those organs affected by diabetes, diabetic nephropathy is noteworthy, as diabetes is the major cause of terminal renal failure in our country and throughout the world. The treatment of diabetic patients with renal failure is challenging, as severe nephropathy leads to metabolic changes that increases the chance of hypoglycemia. Insulin half-life is increased in patients with nephropathy. The development of new oral hypoglycemic agents in the past few years broadened the number of potentially advantageous therapeutic drug
1. Serviço de Diabetes. Hospital Universitário Pedro Ernesto. Universidade do Estado do Rio de Janeiro. Rio de Janeiro, RJ, Brasil.

\author{
*Endereço para correspondência: \\ HUPE, UERJ \\ Boulevard 28 de Setembro, 77 \\ Rio de Janeiro, RJ, Brasil. CEP: 20551-030 \\ E-mail: raquel.abib@ig.com.br
}

Revista HUPE, Rio de Janeiro, 2015;14(4):50-53

doi: $110.12957 /$ rhupe.2015.20057

Recebido em 19/03/2015. Aprovado em 08/12/2015.

options for those patients. On the other hand, the complexity in the individualization of treatment is increased, as it is crucial to know the contraindications, dosage adjustment needed, risk of adverse events and nephrotoxicity of each one of those drugs. In patients in dialysis, various factors can make it difficult to achieve a good glycemic control, including factors that are related to the nephropathy itself or related to the dialysis method used. Another factor that complicates the treatment of these patients is the fact that the laboratory parameters traditionally used for the evaluation of glycemic control are prone to variations caused by the renal failure, reducing their accuracy. The knowledge of those variables is crucial to appropriately individualize the complex treatment of diabetic patients with renal failure, so that the best glycemic control could be achieved with the minimum risk of hypoglycemia.

Keywords: Renal insufficiency; Diabetes mellitus; Therapeutics.

\section{Resumen}

\section{Particularidades en el manejo de la diabetes en pacientes con nefropatía}

La diabetes es una enfermedad sistémica que puede afectar a muchos órganos, con reducción de la calidad y esperanza de vida. Entre los órganos afectados, la nefropatía diabética merece ser destacada; siendo la diabetes la principal causa de la enfermedad renal terminal en Brasil y en todo el mundo. El tratamiento de los pacientes diabéticos con insuficiencia renal es un gran desafío clínico, ya que la presencia de la nefropatía severa conduce a cambios metabólicos que aumentan el riesgo de hipoglucemia. Hay aumento de la vida media de 
la insulina en los pacientes con nefropatía. El desarrollo de nuevos hipoglucemiantes orales en los últimos años amplió las opciones terapéuticas potencialmente ventajosas para este grupo de pacientes. Por otra parte, la complejidad de la individualización del tratamiento se vuelve aún mayor, ya que es fundamental conocer las contraindicaciones, ajustes de la posología necesarios, riesgos de efectos secundarios y nefrotoxicidad de cada uno de estos medicamentos. En los pacientes en diálisis, varios factores pueden afectar negativamente el control glucémico, incluyendo tanto factores relacionados con la nefropatía como relacionados con el método de diálisis utilizado. Otro factor que contribuye a

\section{Introdução}

O diabetes é a principal causa de doença renal terminal no Brasil e no mundo. A alteração do metabolismo da insulina e de hipoglicemiantes e a redução da gliconeogênese na insuficiência renal aumentam a susceptibilidade à hipoglicemia. A maior propensão à hipoglicemia, a diminuição da acurácia dos principais métodos de avaliação do controle glicêmico e a escassez de estudos randomizados com esse grupo de pacientes fazem do tratamento da hiperglicemia em nefropatas um desafio na prática clínica.

\section{Controle glicêmico em pacientes com nefropatia}

Sabe-se que o controle glicêmico intensivo reduz o desenvolvimento de micro e macroalbuminúria, tanto no diabetes tipo 1 quanto no tipo $2 .^{1-3}$ No entanto, as evidências de que o controle intensivo previne o desenvolvimento de doença renal terminal são limitadas. Também não há estudos randomizados avaliando os benefícios do controle glicêmico no grupo de pacientes diabéticos que já apresentam doença renal avançada. Por outro lado, uma metanálise recente de estudos observacionais com pacientes diabéticos em hemodiálise demonstrou que níveis de $\mathrm{HbA1c} \geq 8,5 \%$ estão associados a uma maior taxa de mortalidade quando comparados com níveis entre 6,5 e 7,4\%. ${ }^{4}$ Outro estudo observacional com 2.798 diabéticos em diálise peritoneal demonstrou aumento da mortalidade em pacientes com HbA1C acima de $8 \%$ quando comparados com aqueles com HbA1c abaixo de $8 \%{ }^{5}$

Na prática clínica, o alvo de hemoglobina glicada em pacientes com doença renal crônica (DRC) deve ser individualizado de acordo com o risco de hipoglicemia, expectativa de vida e comorbidades. ${ }^{6,7}$ De forma geral, recomenda-se como meta de tratamento HbA1C próxima a 7\% para nefropatas sem disfunção grave e < la complejidad del tratamiento de estos pacientes es que los parámetros de laboratorio, utilizados tradicionalmente para evaluar el control glucémico, sufren influencias importantes como resultado de la enfermedad renal terminal, con posible reducción de su exactitud. El conocimiento de todos estos factores es esencial, para que el complejo tratamiento de los pacientes diabéticos con enfermedad renal terminal, pueda ser individualizado adecudamente, con el fin de obtener el mejor control glucémico posible con el mínimo riesgo de hipoglucemia.

Palabras clave: Insuficiencia renal; Diabetes mellitus; Terapéutica.

7,5-8\% para aqueles com doença renal avançada (taxa de filtração glomerulara (TFG) $<10 \mathrm{ml} / \mathrm{min} / 1.73 \mathrm{~m}^{2}$ ) ou em terapia dialítica. ${ }^{7.8}$

A acurácia da HbA1c em refletir o controle glicêmico pode estar comprometida em pacientes com DRC. Valores de HbA1c que subestimem os níveis glicêmicos podem ser detectados devido à diminuição da meia-vida de hemácias, transfusões, tratamento com análogo de eritropoietina e hemólise. Valores falsamente elevados também podem ser encontrados quando a metodologia de dosagem da HbA1C é a cromatografia de alta performance (HPLC), devido à carbamilação da hemoglobina, que ocorre na presença de elevação dos níveis de ureia. Já a dosagem de HbA1C por ensaio imunoturbidimétrico não é afetada por níveis elevados de ureia. ${ }^{9}$ Quando este método é utilizado, os principais fatores que interferem no nível de HbA1C são a presença de anemia e a terapia com análogo de eritropoietina. $\mathrm{Na}$ anemia por doença renal avançada, a meia-vida das hemácias está diminuída e o menor tempo de exposição ao ambiente hiperglicêmico leva à menor taxa de glicação. Os análogos de eritropoietina aumentam o número de eritrócitos imaturos na circulação, os quais são menos propensas à glicação. Portanto, quando métodos mais atuais de dosagem são utilizados, a tendência é de que os níveis de HbA1c subestimem os níveis de glicemia em pacientes com doença renal avançada. ${ }^{10,11}$

A frutosamina também não parece ser um bom marcador de controle glicêmico em nefropatas. A albumina glicada, que reflete os níveis glicêmicos das duas últimas semanas, poderia ser uma alternativa. Estudos sugerem que a albumina glicada seja superior à HbA1c em refletir os níveis glicêmicos e em predizer a taxa de hospitalização e a sobrevida em pacientes com insuficiência renal. ${ }^{12,13}$

Em pacientes em diálise, vários fatores podem prejudicar o controle glicêmico, como a anorexia associada 


\section{Artigo de revisão}

à uremia, redução da depuração da insulina, redução da absorção intestinal da glicose e fatores relacionados à diálise. Durante a diálise peritoneal, o fluido utilizado é absorvido sistemicamente em volume significativo e a dextrose nele contida pode levar à hiperglicemia. Uma alternativa para evitar o aumento da glicemia é a substituição da dextrose por icodextrina, que é minimamente absorvida pela membrana peritoneal. A utilização da icodextrina tem como desvantagem a superestimação dos níveis glicêmicos por glicosímetros, que utilizam a enzima glicose desidrogenase pirroquinolinequinona. ${ }^{14}$ Outra possibilidade é a administração de insulina intraperitoneal, que parece possibilitar melhor controle glicêmico, mas pode causar complicações graves, limitando sua indicação. ${ }^{8}$

\section{Princípios do tratamento do diabetes em pacientes com nefropatia}

Pacientes que iniciam hemodiálise (HD) frequentemente necessitam de menores doses de insulina, devido à diminuição da resistência insulínica e à redução do metabolismo da insulina pela perda da função renal. Episódios de hipoglicemia são mais comuns em pacientes em $\mathrm{HD}$, o que pode ser minimizado com o uso de soluções dialíticas com elevada concentração de glicose e uso de doses de insulina diferentes nos dias de realização de HD.

A insulina frequentemente é o agente antidiabético mais seguro na presença de DRC grave. No entanto, atualmente dispõe-se de novos fármacos efetivos e bem-tolerados que podem ser uma melhor opção para alguns desses pacientes. $\mathrm{O}$ uso de hipoglicemiantes em nefropatas pode estar associado a um maior risco de hipoglicemia e outros efeitos colaterais, ou ainda à redução da eficácia do agente. ${ }^{9}$ A tabela 1 resume as

Tabela 1. Uso de hipoglicemiantes na doença renal crônica (DRC) estágios 3-5. Adaptada de Williams ME e Garg R. ${ }^{9}$

\begin{tabular}{|c|c|c|c|}
\hline Classe & Agentes & Riscos na DRC & $\begin{array}{c}\text { Ajustes de doses / } \\
\text { contraindicações na DRC }\end{array}$ \\
\hline Sulfonilureias & $\begin{array}{l}\text { Glibenclamida } \\
\text { Glipizida } \\
\text { Glimepirida } \\
\text { Gliclazida }\end{array}$ & Hipoglicemia & $\begin{array}{l}\text { - Usar com cautela, iniciar doses baixas } \\
\text { - Evitar glibenclamida } \\
\text { - Preferir glipizida (metabolismo hepático) }\end{array}$ \\
\hline Biguanidas & Metformina & Acidose láctica & $\begin{array}{l}\text { - Reduzir dose se TFG }<45 \mathrm{~mL} / \mathrm{min} / 1,73 \mathrm{~m} 2 \\
\text { - Contraindicada se TFG }<30 \mathrm{~mL} / \mathrm{min} / 1,73 \mathrm{~m} 2\end{array}$ \\
\hline Metiglinidas & $\begin{array}{l}\text { Repaglinida } \\
\text { Nateglinida }\end{array}$ & Hipoglicemia & $\begin{array}{l}\text { - Mais seguras que sulfonilureias } \\
\text { - Preferir nateglinida } \\
\text { - Se TFG < 30, iniciar doses baixas }\end{array}$ \\
\hline Glitazonas & Pioglitazona & Retenção hídrica & $\begin{array}{l}\text { - Evitar nos pacientes com risco de retenção } \\
\text { hídrica }\end{array}$ \\
\hline $\begin{array}{l}\text { Inibidores da } \\
\text { alfaglicosidase }\end{array}$ & Acarbose & Insuficiência hepática & - Contraindicado se $\mathrm{Cr}$ sérica $>2 \mathrm{mg} / \mathrm{dL}$ \\
\hline Inibidores da DPP4 & $\begin{array}{l}\text { Sitagliptina } \\
\text { Vildagliptina } \\
\text { Saxagliptina } \\
\text { Alogliptina } \\
\text { Linagliptina }\end{array}$ & $\begin{array}{l}\text { Bem tolerados, } \\
\text { porém faltam estu- } \\
\text { dos de longo prazo }\end{array}$ & $\begin{array}{l}\text {-Sitagliptina: TFG } 30-50 \mathrm{~mL} / \mathrm{min}: 50 \mathrm{mg} / \mathrm{d} \text {; } \\
\text { TFG < 30ml/min: } 25 \mathrm{mg} / \mathrm{d} \\
\text {-Vildagliptina: se TFG < 50ml/min: } 50 \mathrm{mg} / \mathrm{d} \\
\text { - Saxagliptina: TFG } \leq 50 \mathrm{ml} / \mathrm{min}: 2,5 \mathrm{mg} / \mathrm{d} \\
\text {-Alogliptina: TFG 30-60: 12,5 mg/d; TFG<30 } \\
\mathrm{mL} / \mathrm{min}: 6,25 \mathrm{mg} / \mathrm{d} \\
\text { - Linagliptina (excreção biliar): sem necessi- } \\
\text { dade de ajuste }\end{array}$ \\
\hline $\begin{array}{l}\text { Miméticos e análogos do } \\
\text { GLP-1 }\end{array}$ & $\begin{array}{l}\text { Exenatida } \\
\text { Exenatide de liberação } \\
\text { prolongada } \\
\text { Liraglutida } \\
\text { Lixisenatide }\end{array}$ & $\begin{array}{l}\text { Náusea e vômitos, } \\
\text { possível risco de } \\
\text { injúria renal aguda }\end{array}$ & - Uso não recomendado \\
\hline Inibidores da SGLT2 & $\begin{array}{l}\text { Canagliflozina } \\
\text { Dapagliflozina } \\
\text { Empagliflozina }\end{array}$ & Redução da eficácia & $\begin{array}{l}\text { - Reduzir dose se TFG }<60 \mathrm{~mL} / \mathrm{min} \\
\text { - Contraindicados se TFG }<45 \mathrm{~mL} / \mathrm{min}\end{array}$ \\
\hline
\end{tabular}


principais considerações sobre o uso dos agentes hipoglicemiantes em pacientes com DRC.

A terapia com insulina em pacientes com DRC deve seguir os mesmos princípios utilizados no tratamento de indivíduos sem nefropatia. As doses de insulina tendem a ser menores no paciente com DRC, devido ao aumento no seu tempo de ação. A insulina NPH pode ser administrada uma ou duas vezes ao dia nesses pacientes, assim como a insulina detemir. A glargina também pode apresentar tempo de ação aumentado. ${ }^{9}$ Não há contraindicação para o uso da insulina degludeca na DRC. As insulinas ultrarrápidas estão associadas a um menor risco de hipoglicemia do que a insulina regular, especialmente em pacientes com DRC. Em casos selecionados, o uso do sistema de infusão contínua de insulina pode ser vantajoso.

\section{Conclusão}

O tratamento do diabetes no paciente com DRC é complexo e necessita ser individualizado. Estudos randomizados com indivíduos com diferentes graus de DRC são necessários para que se amplie o conhecimento sobre as particularidades do manejo do diabetes nesses pacientes.

\section{Referências}

1. Intensive blood-glucose control with sulphonylureas or insulin compared with conventional treatment and risk of complications in patients with type 2 diabetes (UKPDS 33). UK Prospective Diabetes Study (UKPDS) Group. Lancet. 1998 Sep 12;352(9131):837-53.

2. Levin SR, Coburn JW, Abraira C, et al. Effect of intensive glycemic control on microalbuminuria in type 2 diabetes. Veterans Affairs Cooperative Study on Glycemic Control and Complications in Type 2 Diabetes Feasibility Trial Investigators. Diabetes care. 2000 Oct;23(10):1478-85.

3. de Boer IH, Sun W, Gao X, et al. Effect of intensive diabetes treatment on albuminuria in type 1 diabetes: long-term follow-up of the Diabetes Control and Complications Trial and Epidemiology of Diabetes Interventions and Complications study. DCCT/EDIC research group. Lancet Diabetes Endocrinol. 2014 Oct;2(10):793-800. doi: 10.1016/S2213-8587(14)70155-X. Epub 2014 Jul 17.

4. Hill CJ, Maxwell AP, Cardwell CR, et al. Glycated hemoglobin and risk of death in diabetic patients treated with hemodialysis: a meta-analysis. Am J Kidney Dis. 2014 Jan;63(1):84-94.

5. Duong U, Mehrotra R, Molnar MZ, et al. Glycemic control and survival in peritoneal dialysis patients with diabetes mellitus. Clin J Am Soc Nephrol. 2011 May;6(5):1041-8.

6. Standards of medical care in diabetes--2014. Diabetes Care. 2014 Jan;37 Suppl 1:S14-80.

7. KDOQI Clinical Practice Guideline for Diabetes and CKD: 2012 Update. Am J Kidney Dis. 2012 Nov;60(5):850-86.

8. Iglesias P, Diez JJ. Insulin therapy in renal disease. Diabetes Obes Metab. 2008 Sep;10(10):811-23.

9. Williams ME, Garg R. Glycemic management in ESRD and earlier stages of CKD. Am J Kidney Dis. 2014 Feb;63(2 Suppl 2):S22-38.

10. Peacock TP, Shihabi ZK, Bleyer AJ, et al. Comparison of glycated albumin and hemoglobin A(1c) levels in diabetic subjects on hemodialysis. Kidney international. 2008 May;73(9):1062-8.

11. Riveline JP, Teynie J, Belmouaz S, et al. Glycaemic control in type 2 diabetic patients on chronic haemodialysis: use of a continuous glucose monitoring system. Nephrol Dial Transplant. 2009 Sep;24(9):2866-71.

12. Inaba M, Okuno S, Kumeda $Y$, et al. Glycated albumin is a better glycemic indicator than glycated hemoglobin values in hemodialysis patients with diabetes: effect of anemia and erythropoietin injection. J Am Soc Nephrol. 2007 Mar;18(3):896-903.

13. Freedman $\mathrm{Bl}$, Andries L, Shihabi ZK, et al. Glycated albumin and risk of death and hospitalizations in diabetic dialysis patients. Clin J Am Soc Nephrol. 2011 Jul;6(7):1635-43.

14. Sloand JA. Dialysis patient safety: safeguards to prevent iatrogenic hypoglycemia in patients receiving icodextrin. Am J Kidney Dis. 2012 Oct;60(4):514-6. 